\title{
Living with COPD: the struggle for breath and for lung volume reduction therapies
}

\author{
Ravi Mahadeva ${ }^{1}$ and Zaid Zoumot ${ }^{2}$
}

Affiliations: ${ }^{1}$ Cambridge COPD Centre, Addenbrookes Hospital, Cambridge, UK. ${ }^{2}$ Respiratory and Critical Care Institute, Cleveland Clinic Abu Dhabi, Abu Dhabi, United Arab Emirates.

Correspondence: Zaid Zoumot, Respiratory and Critical Care Institute, Cleveland Clinic Abu Dhabi, Abu Dhabi, United Arab Emirates. E-mail: zoumotzaclevelandclinicabudhabi.ae

@ERSpublications

Access to lung volume reduction for advanced emphysema http://ow.ly/Iqru30jbvbE

Cite this article as: Mahadeva R, Zoumot Z. Living with COPD: the struggle for breath and for lung volume reduction therapies. ERJ Open Res 2018; 4: 00005-2018 [https://doi.org/10.1183/ 23120541.00005-2018].

Chronic obstructive pulmonary disease (COPD) is an incurable disease of high prevalence and is the third leading cause of death worldwide [1]. The disease is characterised by progressive breathlessness that in advanced cases, causes intractable breathlessness, a significantly impaired quality of life, progressive disability, and increased and premature mortality.

The characteristic obstructive deficit can be due to a variety of anatomical changes in COPD lungs; in particular, pathology in the small airways and loss of alveolar tissue (emphysema). The distinction between the predominant airway form of COPD and emphysema, and their relationship to the patients' symptoms and prognosis takes on extra significance due to recent advances in phenotype-based therapies. Emphysema not only causes impairment in gas exchange but also results in gas trapping and hyperinflation. Emphysematous hyperinflation is thought to contribute to breathlessness through a variety of mechanisms including dynamic hyperinflation, impairment of function of the diaphragm and nonemphysematous lung tissue including suboptimal ventilation-perfusion relationships, increased work of breathing with adverse nutritional effects and accelerated sarcopenia [1,2]. The recognition of this mechanism in the 1950s hatched the concept of reduction of excessive nonfunctioning lung to improve breathlessness and quality of life in emphysema: lung volume reduction (LVR) [3]. The aim of lung volume reduction surgery (LVRS) is to resect the most emphysematous part of the lung, allowing healthier, less compliant areas to function more effectively; thus, reducing hyperinflation, and improving expiratory flow, diaphragmatic function and respiratory muscle mechanics. Fast-forward to 2003, and the publication of the National Emphysema Treatment Trial (NETT) [4]. This landmark study clearly showed that careful patient selection was critical to achieving a good outcome following LVRS; subgroups demonstrated marked benefits to quality of life, exercise capacity and reduced mortality following bilateral LVRS. It is therefore very important to seek out the hyperinflated emphysematous COPD phenotype, as it lends to the potential of life-changing treatment with LVR. It is worth bearing in mind that current mortality following all modalities of LVR is low and the quality of life improvements following LVR in selected patients are of a magnitude greater than with current inhaled therapies [5-9]. Notably, in addition to the well documented improvements in lung function, marked benefit to quality of life (fall in St George's Respiratory Questionnaire score of $>14$ points), with responder rates of $80 \%$ achieving the minimal clinically important difference have been documented following LVR [10]. This relief of symptoms and restoration of function has to be the main goal of LVR. The advent of bronchoscopic techniques to achieve LVR, together with improvements in surgical, anaesthetic and post-operative approaches, have driven forward valuable refinements in patient selection [11-13]. These factors only further emphasise that experience and skill in patient selection and peri-operative management are critical

Received: Jan 072018 | Accepted after revision: March 092018

Copyright (CERS 2018. This article is open access and distributed under the terms of the Creative Commons Attribution Non-Commercial Licence 4.0. 
to determining outcomes following LVR, and have catapulted the field into a specialist and multidisciplinary arena.

There are currently various options either available or in development to achieve LVR: LVRS, endobronchial valves, nitinol coils, thermal vapour and polymer sealant [14]. The aim of the different options is to broaden the eligibility criteria for LVR for individuals with hyperinflation and emphysema whilst maintaining a favourable risk-benefit profile. The added nuance of specialised assessments and various tailored LVR interventions themselves require a multidisciplinary team (MDT) that is experienced in COPD management and LVR techniques. Most expert centres would advocate that the team comprises of a chest physician, thoracic surgeon, thoracic radiologist and respiratory nurse to facilitate careful patient selection to personalise risk and benefits and individualise the most appropriate intervention, and most expert centres would also include an interventional bronchoscopist and COPD physician [12]. The success of this approach is exemplified by the two centres involved in the study by BuTTERY et al. [15], and both teams have recently reported risk assessment tools and excellent outcomes [16, 17].

The report by BUTTERY et al. [15] is novel in the area in that it focuses on the patient perspective. The main finding of patients reporting having to fight for a referral and poor knowledge by healthcare professionals is disappointing, but sadly not out of keeping with feedback received from many individuals. Patients often have to research these treatments themselves, as there is poor knowledge and understanding of outcomes and pathways across the healthcare system. Although with some limitations, the take-home message of the report is in accordance with a previous report of attitudes of healthcare professionals to LVR, the conclusion of which was that most respiratory physicians in the UK were unsure about the risk of death and significantly overestimated risks of LVRS, and that there was patchy access to a MDT to discuss management of patients with advanced COPD [18]. It should perhaps not be a surprise that the need to concentrate LVR procedures and expertise to a limited number of highly specialised centres comprising all of the above mentioned individuals means that there is poor geographical coverage throughout countries, contributing to a lack of understanding and consideration for LVR amongst the primary and secondary care communities. For example, in the USA, only 26 centres are approved by the Centres for Medicare and Medicaid Services to perform LVRS, leaving gaps in access to LVR. This perpetuates a cycle of reduced knowledge, lower referrals and ultimately fewer interventions being performed. Accessible international data for numbers of LVRS procedures being performed is limited to those from the UK, USA and Japan, and numbers are likely to be even lower in other countries: in the UK, only 164 LVRS procedures were undertaken in 2014-2015 in the whole of the National Health Service and Ireland (with only three deaths) [19]. Similarly, in the 10 years after NETT, only 3307 patients had LVRS in the USA according to the Nationwide Inpatient Sample database, with a trend of declining annual numbers and just over 200 LVRS procedures in 2010; and in Japan in 2013, a total of 24 LVRS procedures were performed (omitting bullectomies) [20,21].

There are no publicly accessible data on the use of endobronchial valves or nitinol coils in the UK or elsewhere; however, manufacturers of the implants now report procedures numbering in the several thousand throughout the world. Germany has led the way due to a favourable system for funding novel therapies for advanced diseases in the absence of alternative treatment options, and bronchoscopic LVR now has a designated reimbursement code. Endobronchial valves and/or coils are also reimbursed in the Netherlands, UK, Austria, Switzerland, Turkey, Italy, Australia and United Arab Emirates amongst various other countries, using standard or special reimbursement codes. There is no funding for bronchoscopic LVR procedures in the Far East at present and there is no US Food and Drug Administration (FDA) approval for bronchoscopic LVR techniques, which remain restricted for use in clinical trials in the USA. The UK National Institute for Health and Care Excellence (NICE) has updated its recommendations, which now state that "current evidence on the safety and efficacy of endobronchial valve insertion to reduce lung volume in emphysema is adequate in quantity and quality to support the use of this procedure provided that standard arrangements are in place for clinical governance, consent and audit" [12]. The LIBERATE study (www.clinicaltrials.gov identifier number NCT01796392), an FDA investigational device exemption approved randomised controlled trial of endobronchial valves compared with best medical care, has completed data collection for the primary endpoint measures and results are anticipated in early 2018. If the primary outcomes are met, this should lead to FDA approval.

Data on the size of the patient pool potentially suitable for LVR are scarce but it is estimated that $\sim 15 \%$ of emphysema patients are eligible for LVRS on physiological and computed tomography appearances [22]. However, with the advent of bronchoscopic techniques and the identification of clinical phenotypes most responsive to the various LVR techniques, the patient pool eligible is likely to be much larger. Recent data indicating that there are $~ 1.2$ million people currently diagnosed with COPD in the UK [23] give a strong indication that there is major underutilisation of LVR. 
Why is it that LVR options are not considered as part of the standard assessment for COPD just as one would consider inhaled therapies and pulmonary rehabilitation? The answer is undoubtedly complex, including possible factors such as undue focus on forced expiratory volume in $1 \mathrm{~s}$ in COPD assessments, inappropriate overestimation of the risks of LVR, lack of robust local pathways and insufficient services. The situation is compounded by the nihilism in COPD whereby many patients are not considered for LVR by primary and secondary care physicians, and are often discharged from follow-up with the impression that nothing further can be done [9]. As funding for bronchoscopic LVR is now available in many countries, it is likely that the number of bronchoscopic LVR procedures will continue to increase year on year and outstrip numbers of LVRS procedures being performed. This in itself should disseminate knowledge to healthcare professionals and patients alike about all forms of LVR, and hopefully drive referrals, but more crucially, it may remove some of the obstacles faced by patients who should be considered for therapies that may significantly improve their quality of life, without the need to "fight" for that to which they are entitled.

The report from BuTtery et al. [15] is timely, serving to challenge healthcare systems to act; clearly, further work is needed to understand and to develop pathways from primary care through to specialist centres. Patient empowerment, further developments in patient selection and additional health economic data are critical for further progress. Moreover, healthcare providers need to recognise the clinical need and benefits of such interventions to drive organised systems of care and assessments to support patients appropriately. This report on behalf of patients sends an important message to providers, commissioners and clinicians: LVR offers the prospect of meaningful benefits to more patients with COPD; what all can agree upon is that patients are given an equal opportunity to be eligible for appropriate treatments.

Conflict of interest: R. Mahadeva has participated in educational activities and advisory boards between 2013 and 2018 sponsored by AstraZeneca, Boehringer Ingelheim, Chiesi, CSL Behring, Kamada, Nutricia, Pfizer and Pulmonx. R. Mahadeva has no real or perceived conflicts of interest in relation to this editorial.

\section{References}

1 Global Initiative for Chronic Obstructive Lung Disease. Global Strategy for the Diagnosis, Management, and Prevention of Chronic Obstructive Pulmonary Disease. http://goldcopd.org/wp-content/uploads/2017/11/GOLD2018-v6.0-FINAL-revised-20-Nov_WMS.pdf

2 Brusasco V, Martinez F. Chronic obstructive pulmonary disease. Compr Physiol 2014; 4: 1-31.

3 Brantigan OC, Mueller E. Surgical treatment of pulmonary emphysema. Am Surg 1957; 23: 789-804.

4 Fishman A, Martinez F, Naunheim K, et al. A randomized trial comparing lung-volume-reduction surgery with medical therapy for severe emphysema. N Engl J Med 2003; 348: 2059-2073.

5 van Agteren JEM, Carson KV, Tiong LU, et al. Lung volume reduction surgery for diffuse emphysema. Cochrane Database Syst Rev 2016; 10: CD001001.

6 Criner GJ, Sternberg AL. National Emphysema Treatment Trial: the major outcomes of lung volume reduction surgery in severe emphysema. Proc Am Thorac Soc 2008; 5: 393-405.

7 Pietzsch JB, Garner A, Herth FJF. Cost-effectiveness of endobronchial valve therapy for severe emphysema: a model-based projection based on the VENT study. Respiration 2014; 88: 389-398.

8 Ramsey SD, Shroyer AL, Sullivan SD, et al. Updated evaluation of the cost-effectiveness of lung volume reduction surgery. Chest 2007; 131: 823-832.

9 Zoumot Z, Jordan S, Hopkinson NS. Emphysema: time to say farewell to therapeutic nihilism. Thorax 2014; 69: 973-975.

10 Klooster K, ten Hacken NHT, Hartman JE, et al. Endobronchial valves for emphysema without interlobar collateral ventilation. N Engl J Med 2015; 373: 2325-2335.

11 Rathinam R, Oey I, Steiner M, et al. The role of the emphysema multidisciplinary team in a successful lung volume reduction surgery programme. Eur J CardioThorac Surg 2014; 46: 1021-1026.

12 National Institute for Health and Care Excellence. Endobronchial valve insertion to reduce lung volume in emphysema. https://www.nice.org.uk/guidance/ipg600

13 National Institute for Health and Care Excellence. Lung volume reduction surgery for advanced emphysema. https:/www.nice.org.uk/guidance/ipg114/resources/lung-volume-reduction-surgery-for-advanced-emphysema-pdf1899863038265029

14 Shah PL, Herth FJ, van Geffen WH, et al. Lung volume reduction for emphysema. Lancet Respir Med 2017; 5: 147-156.

15 Buttery S, Lewis A, Oey I, et al. Patient experience of lung volume reduction procedures for emphysema: a qualitative service improvement project. ERJ Open Res 2017; 3: 00031-2017.

16 Clark SJ, Zoumot Z, Bamsey O, et al. Surgical approaches for lung volume reduction in emphysema. Clin Med (Lond) 2014; 14: 122-127.

17 Greening NJ, Vaughan P, Oey I, et al. Individualised risk in patients undergoing lung volume reduction surgery: the Glenfield BFG score. Eur Respir J 2017; 49: 1601766.

18 McNulty W, Jordan S, Hopkinson NS. Attitudes and access to lung volume reduction surgery for COPD: a survey by the British Thoracic Society. BMJ Open Respir Res 2014; 1: e000023.

19 Society for Cardiothoracic Surgery in Great Britain and Northern Ireland. Outcomes: Thoracic. https://scts.org/ outcomes/thoracic/

20 Ahmad S, Taneja A, Kurman J, et al. National trends in lung volume reduction surgery in the United States: 2000 to 2010. Chest 2014; 146: e228-e229. 
21 Committee for Scientific Affairs, The Japanese Association for Thoracic Surgery, Masuda M, et al. Thoracic and cardiovascular surgery in Japan during 2013: annual report by The Japanese Association for Thoracic Surgery. Gen Thorac Cardiovasc Surg 2015; 63: 670-701.

22 Akuthota, P, Litmanovich D, Zutler M, et al. An evidence-based estimate on the size of the potential patient pool for lung volume reduction surgery. Ann Thorac Surg 2012; 94: 205-211.

23 British Lung Foundation. Chronic obstructive pulmonary disease (COPD) statistics. https://statistics.blf.org.uk/ copd 